\title{
Surgical Treatment of Inadvertent Internal Carotid Artery Lesion by Extraintracranial High-flow Bypass. A Case Report and Review of the Literature
}

\author{
Dilok Tantongtip ${ }^{1} \quad$ Alessia Fratianni $^{2}$ Jost Jenkner ${ }^{2}$ Sebastian Arnold ${ }^{3}$ Uwe Spetzger ${ }^{2}$ \\ ${ }^{1}$ Department of Neurosurgery, Thammasat University, Pathum Thani, \\ Thailand \\ 2 Department of Neurosurgery, Städtisches Klinikum Karlsruhe, \\ Karlsruhe, Germany \\ ${ }^{3}$ Department of Radiology, Städtisches Klinikum Karlsruhe, \\ Karlsruhe, Germany \\ Address for correspondence Dilok Tantongtip, MD, Department of \\ Neurosurgery, 95 Phaholyothin Road, Khlong Nung, Khlong Luang \\ District, Pathum Thani, 12120, Thailand \\ (e-mail: dilok-t@hotmail.com). \\ J Neurol Surg Rep 2015;76:e100-e104.

\begin{abstract}
Keywords

- internal carotid artery injury

- extraintracranial bypass

- transsphenoidal surgery

- pituitary surgery

Internal carotid artery (ICA) injury following transsphenoidal surgery is a rare but potentially fatal complication. Usually, endovascular occlusion of the ICA or stent graft placement is the treatment of these vascular complications described in literature. We present a case of ICA perforation during transsphenoidal surgery in a patient with limited collateral cerebral blood flow and with ectasia of the ICA that rule out an endovascular treatment. We report the surgical revascularization via high-flow extraintracranial radial artery bypass and consicutive artery ligation.
\end{abstract}

\section{Introduction}

The usual approach for surgical resection of pituitary tumors is the transsphenoidal approach, and serious vascular complications are rare. Ciric et al reported a low surgical mortality rate $<1 \%$ and showed a rate of internal carotid artery (ICA) injuries of $1.1 \%$ of all listed complications in transsphenoidal surgery. ${ }^{1,2}$ However, ICA perforation can be lethal. In principle, there is no standard management for these vascular complications; however, the usual surgical strategy in the case of inadvertent ICA injury is immediate compression by packing the sphenoid sinus with different materials to stop the arterial bleeding temporarily. Definitive treatment in the vast majority of reported cases is endovascular treatment, with the aim of either vessel occlusion, which needs the presence of a good collateral blood flow, or coverage of the injured segment with a fully occlusive stent. $^{3}$

In this report we describe a case of ICA injury during transsphenoidal surgery in a patient for whom endovascular treatment was not considered possible given the equipment available.

\section{Case Report}

A 78-year-old female patient presented with slowly progressive visual loss and a bitemporal hemianopsia. Magnetic resonance imaging (MRI) revealed a large sellar mass with suprasellar extension compatible with pituitary macroadenoma ( - Fig. $\mathbf{1}$ ). Laboratory analysis showed it to be a nonfunctioning adenoma. Microsurgical resection of the pituitary adenoma via a transnasal-transsphenoidal
License terms

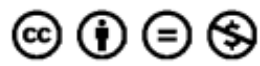

received

May 30, 2014

accepted after revision

January 7, 2015

published online

May 15, 2015
DOI http://dx.doi.org/

10.1055/s-0035-1551670. ISSN 2193-6366. (c) 2015 Georg Thieme Verlag KG Stuttgart · New York 


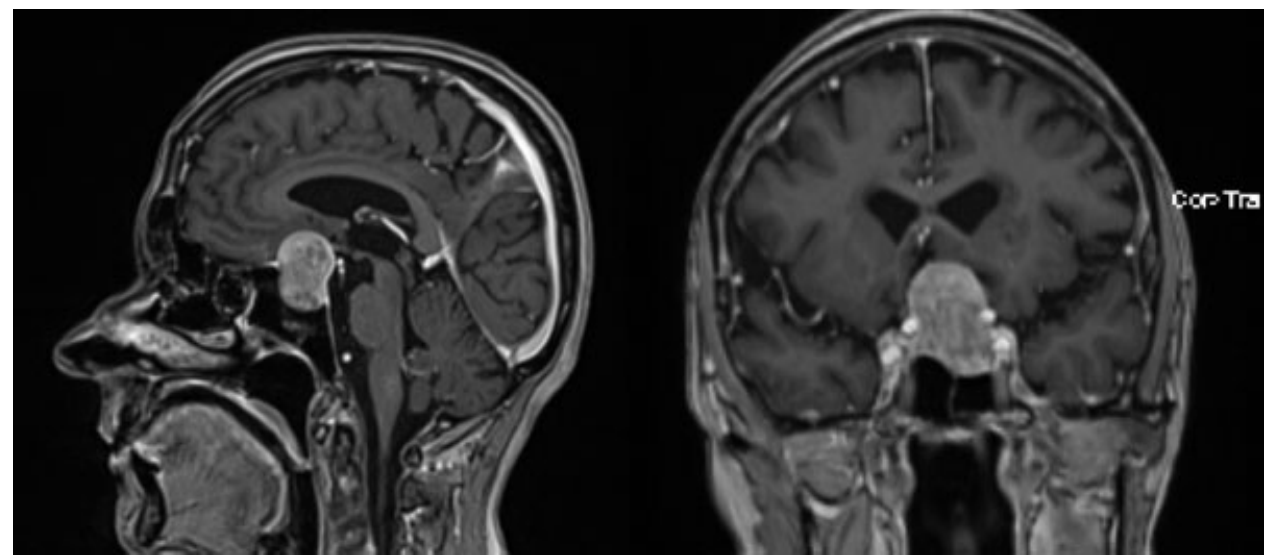

Fig. 1 Sagittal and coronal view magnetic resonance imaging with gadolinium contrast enhancement revealed a sellar mass with suprasellar extension compatible with pituitary adenoma.

approach under image guidance was performed. During removal of the lateral bony part of the floor of the sella with a Kerrison rongeur, an accidental injury of the right ICA occurred with severe arterial bleeding. The bleeding was stopped immediately by packing with Surgicel, fibrin glue, hemostatic matrix (FloSeal, Baxter, Zurich, Switzerland), and autologous fat. The surgical procedure was stopped without removal of the tumor. Postoperatively, the patient underwent emergency cerebral angiography that showed only minimal extravasation of contrast from the cavernous segment of the right ICA (-Fig. 2, arrows). Interestingly, angiography also demonstrated an incidental left ICA bifurcation aneurysm (-Fig. 2) that was not detected at preoperative MRI, and aplasia of the left A1 segment of the anterior cerebral artery. Two days later, when there was no further bleeding from the surgical site, the patient underwent a left pterional approach with removal of the adenoma. At the same time, the unruptured left ICA aneurysm was clipped. During the operation the patient had no bleeding from the right ICA or any epistaxis. The postoperative MRI revealed complete removal of the pituitary adenoma and the absence of residual aneurysm neck (-Fig. 3).
At this stage, the endovascular options for treatment of the injured right cavernous segment of the ICA were discussed with our endovascular colleagues who proposed the use of a flow-diverter stent to cover the injured segment of the right ICA. Unfortunately, the ectatic conformation of the cavernous segment of the right ICA (maximum diameter $>5 \mathrm{~mm}$ ) mismatched the available stent's diameter, and our endovascular colleagues were reluctant to proceed with endovascular treatment. The other reason was that the flow-diverter stent did not have an immediate effect of occlusion of the ICA lesion and would require dual antiplatelet therapy. Another option for endovascular treatment was a covered stent, but there was concern that the stiffness of the covered stent could possibly cause more injury to the vessel. One week after the removal of the nasal packing, the patient regained consciousness, could follow simple commands, and was then extubated. It was assumed that the patient had insufficient collateral cerebral blood flow to allow for complete endovascular occlusion of the right ICA due to hypoplasia of the left A1 segment and very small right posterior communicating artery. Surgical treatment was planned with an extraintracranial high-flow bypass and subsequent occlusion of the injured right ICA. However, the day before scheduled bypass
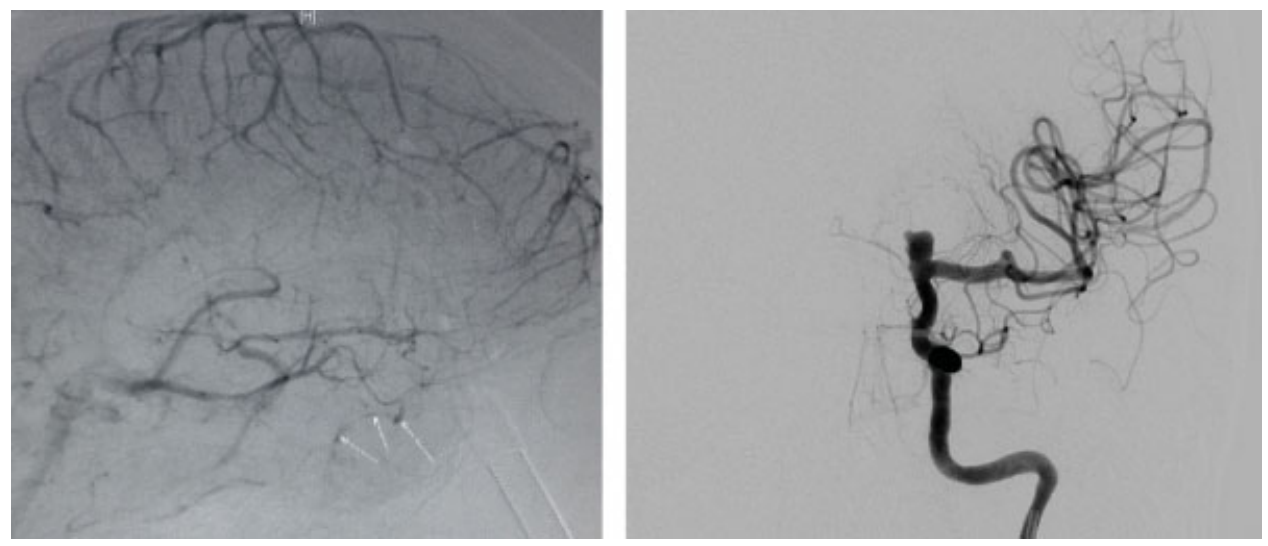

Fig. 2 (Left) Angiographic image of right internal carotid artery (ICA) (venous phase) showed only minimal contrast extravasation (arrows). (Right) Angiographic image of left ICA showed ICA bifurcation aneurysm with A1 segment hypoplasia. 


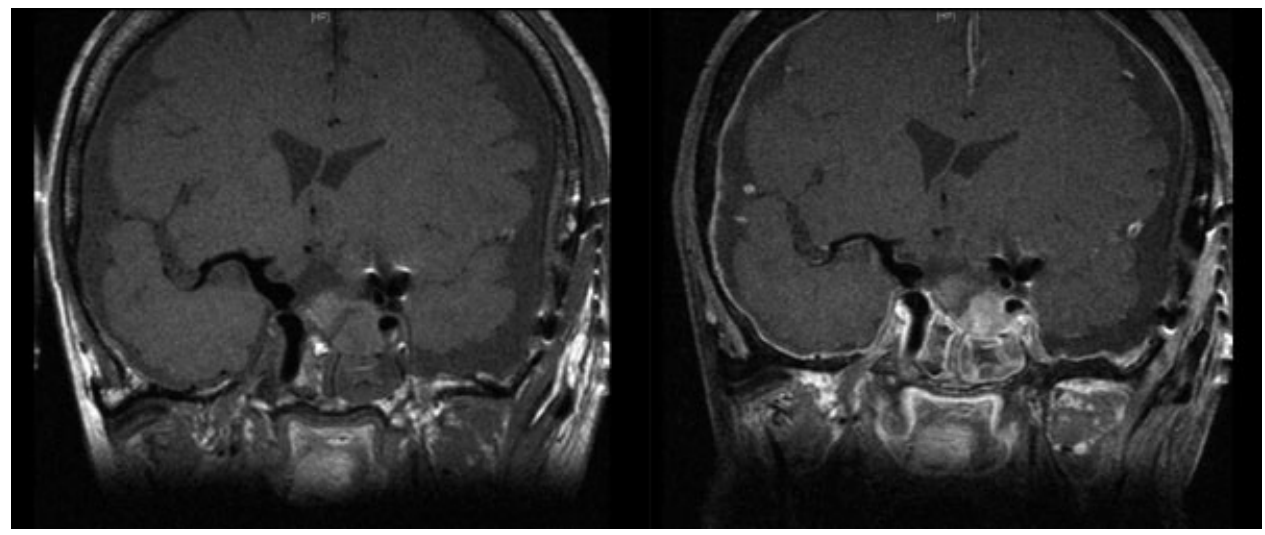

Fig. 3 Postoperative magnetic resonance imaging shows complete removal of the pituitary adenoma and clipping of the left internal carotid artery (ICA) aneurysm with slight contrast enhancement of the packing material. The right ICA demonstrates no lesion or pseudoaneurysm.

surgery, a massive epistaxis occurred during routine cleaning and suction of the airways. An emergency endonasal/transsphenoidal approach with repacking was performed, and the bleeding was stopped successfully. On the same day we proceeded with the planned surgery to bypass the lesion of the right ICA.

Via right pterional craniotomy and opening of the Sylvian fissure, the right ICA, A1 segment of the anterior cerebral artery, and M1 segment of the middle cerebral artery were dissected. A 17-cm-length graft of the radial artery was harvested by the vascular surgeons. In routine fashion the cervical vessels were prepared and the common carotid artery, external carotid artery (ECA), and ICA were exposed. The graft tunnel was planned from the right temporal base, through the infratemporal fossa, to the neck. Using the trajectory planning mode of the navigation system, a small burr hole was drilled in the temporal base. Then a plastic tube (diameter: $7 \mathrm{~mm}$ ) was used as a path for the radial artery graft. The tube was pushed upward from the cervical approach through the burr hole into the temporal fossa. The radial artery was then inserted into the plastic tube and was applied up from the neck through the plastic tube

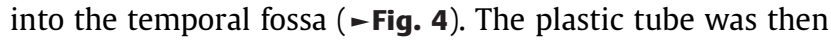
removed.

First, the proximal anastomosis was done on the ECA just distal to the cervical carotid bifurcation (-Fig. 4). In an endto-side anastomosis, the radial artery was sutured to the ECA. Adequate blood flow in the radial artery was checked under the microscope and with Doppler sonography. Second, the optimal position for the distal anastomosis intracranially was selected. The anatomical situation was complicated due to the calcified proximal ICA, so the segment between the ICA bifurcation and the posterior communicating artery was chosen. Temporary clips were placed distal to the posterior communicating artery and proximal to the ICA bifurcation. Doppler sonography demonstrated a slight cross-flow from the contralateral A1. The choroidal artery was not perfused for 45 minutes during the temporary clipping of the supraclinoid ICA. The temporary clips were removed, and the radial artery bypass demonstrated good flow. Finally, the cervical ICA was ligated just distal to the bifurcation (-Fig. 5). The intracranial anastomosis was checked with intraoperative Doppler sonography. Adequate blood flow via the radial artery graft through the anastomosis,

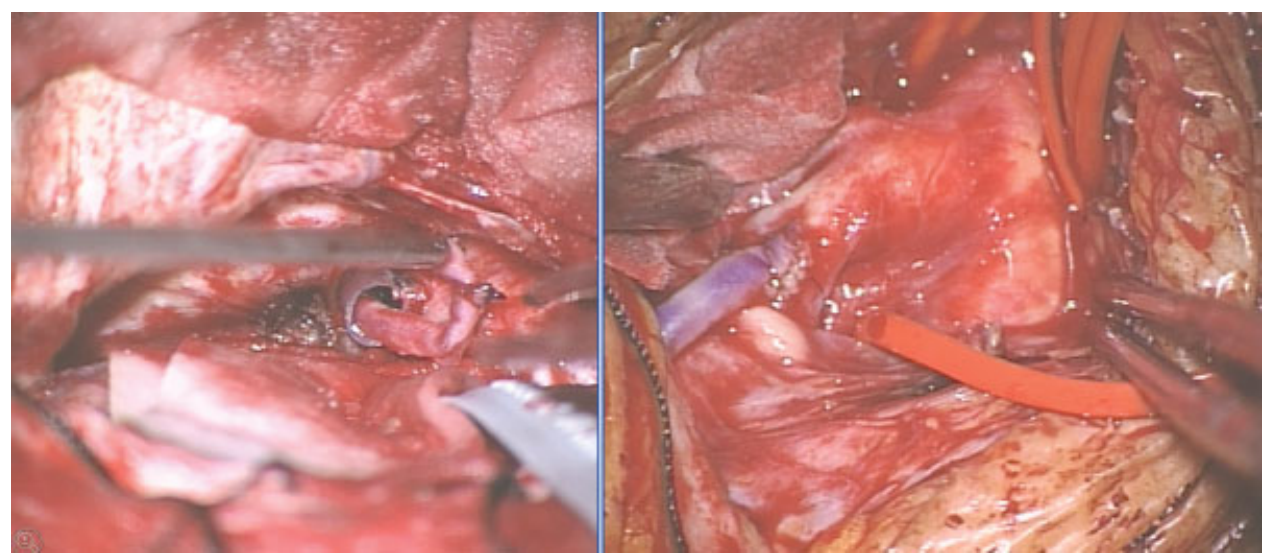

Fig. 4 (Left) Intraoperative views showing the radial artery graft in the plastic tube serve as a guideway from the temporal base through the infratemporal fossa down to the neck. (Right) View into the surgical opening of the neck with the proximal anastomosis of the radial artery to the external carotid artery just distal to the carotid bifurcation. 


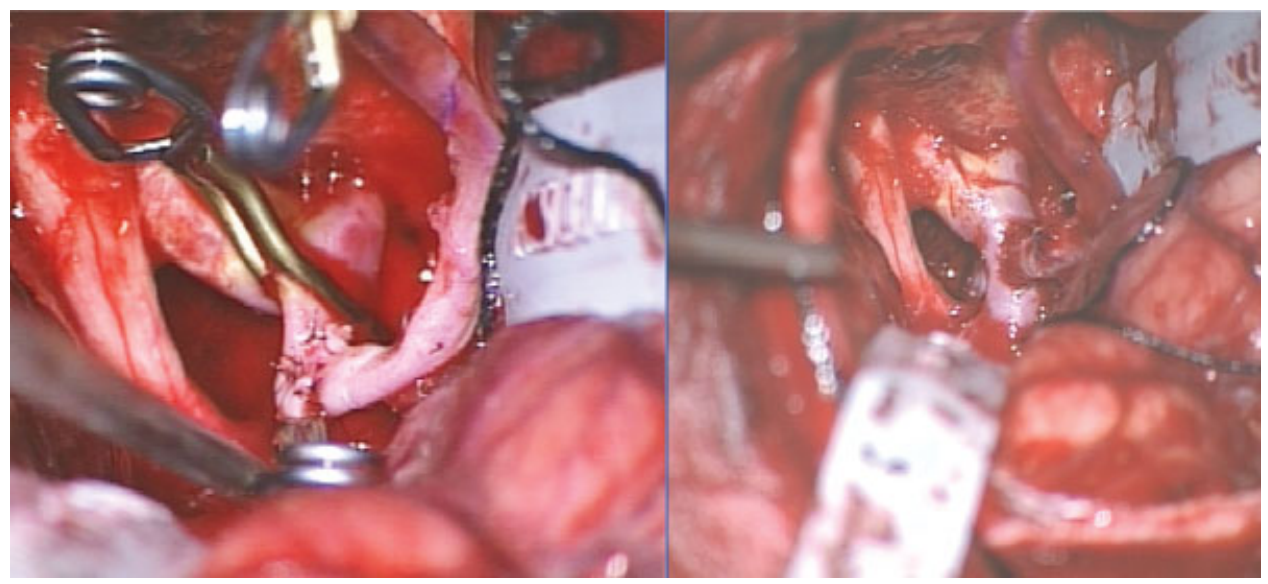

Fig. 5 (Left) Distal anastomosis radial artery to the supraclinoid internal carotid artery (ICA) with temporary clips on the ICA and radial artery graft. (Right) Removal of the temporary clips demonstrated a good blood flow from the radial graft to the ICA.
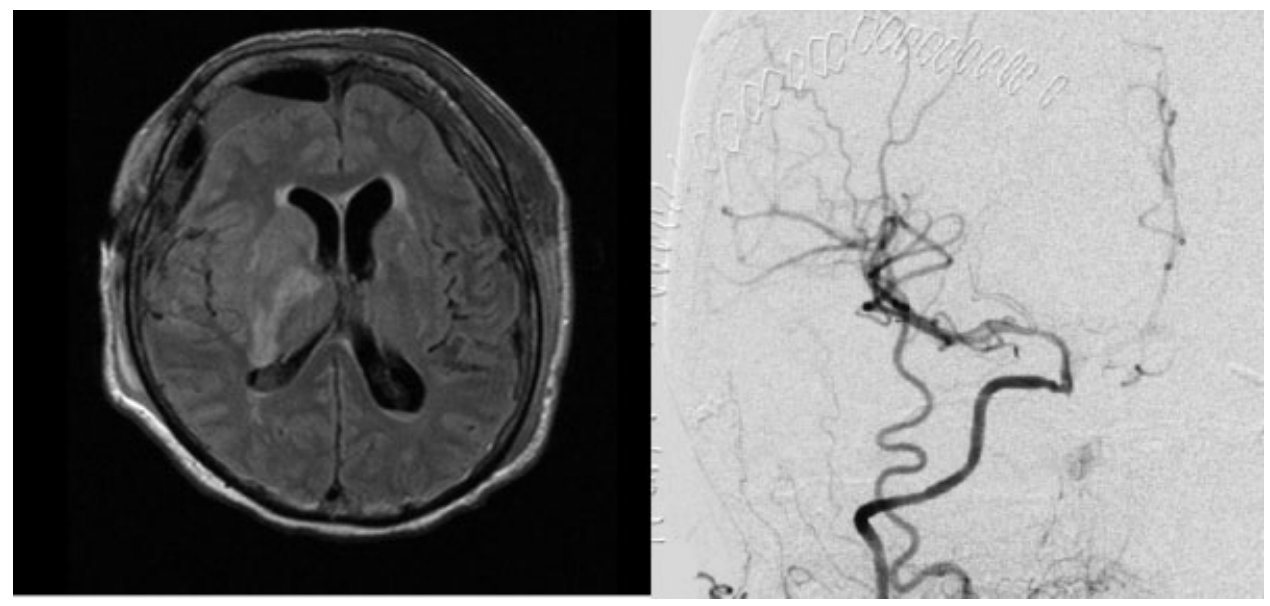

Fig. 6 (Left) Postoperative magnetic resonance imaging showed right infarction of the anterior choroidal artery territory due to prolong ischemic time during bypass surgery. (Right) Postoperative angiogram showed the distal anastomosis (arrow) with good blood flow to the internal carotid artery.

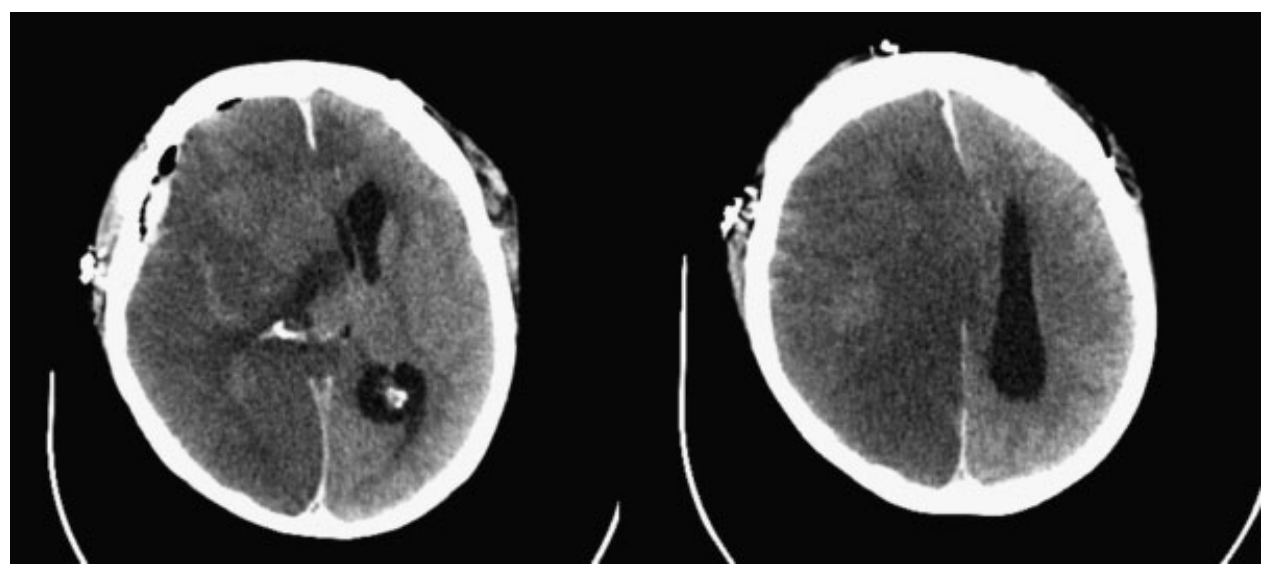

Fig. 7 Computed tomography scan after acute neurologic deterioration (7 days after bypass surgery) showed complete infarction of right hemisphere with herniation. 
into the ICA, A1, and M1, and also to the posterior communicating artery was documented.

Postoperative MRI 1 day after the bypass surgery showed an infarction of the right anterior choroidal artery territory due to the long occlusion time. The cerebral angiography confirmed a patent anastomosis and good flow in the right middle cerebral artery and anterior cerebral artery territories (-Fig. 6). Postoperatively the patient was alert and had an incomplete hemiparesis and was mobilized during the next few days.

However, 1 week postoperatively, the patient developed acute neurologic deterioration with right pupil dilatation. A computed tomography scan showed complete infarction of the right cerebral hemisphere ( - Fig. 7 ), and unfortunately she died 2 days later.

\section{Discussion}

Transsphenoidal surgery is considered to be a safe and effective surgical procedure. Nevertheless life-threatening complications can occur, and carotid injury is a potentially fatal complication. Carotid perforation can cause fatal extravasation, carotid-cavernous fistula, occlusion, and false aneurysm formation, as reported in the literature. ${ }^{4-6}$ Dusick et al proposed the use of Doppler probe and micro-hook blades to avoid carotid injury in transsphenoidal surgery. ${ }^{7}$

There are no universal guidelines for the management of internal carotid perforation. In most patients, bleeding is stopped successfully with aggressive intraoperative packing. The definitive treatment to prevent rebleeding tends to be endovascular. Two intervention strategies have been reported including the occlusion of the ICA, which needs adequate cross-flow, or the implant of a stent graft to cover the ICA's pathologic segment. ${ }^{5,8-10}$ In this report the patient had limited endovascular options because collateral blood flow was thought to be insufficient and the cavernous segment of the ICA was ectatic, preventing the implant of a stent. In this rare condition, surgical treatment with an extracranial-intracranial bypass (EC-IC bypass) and ICA trapping can be considered. In the literature a successful trapping of the ICA with high-flow EC-IC bypass surgery on a patient with an iatrogenic ICA false aneurysm was reported. ${ }^{11}$ Most of the cases reported in the literature showed that intraoperative packing is not sufficient, and complications such as massive epistaxis, false aneurysms, fistulae, or carotid occlusion may occur. $^{4,5}$ According to these data, a definitive treatment should be performed after the packing.
Even if the ideal approach to carotid injuries is endovascular, some patients may not be eligible due to anatomical and technical limitations; therefore other approaches such as internal carotid trapping with high flow EC-IC bypass can be effective. Nevertheless, bypass surgery needs meticulous planning with detailed angiographic imaging, qualitative measurement of collateral blood flow, adequate surgical equipment, and a well-prepared professional team.

\section{References}

1 Ciric I, Ragin A, Baumgartner C, Pierce D. Complications of transsphenoidal surgery: results of a national survey, review of the literature, and personal experience. Neurosurgery 1997;40(2): 225-236; discussion 236-237

2 Sudhakar N, Ray A, Vafidis JA. Complications after trans-sphenoidal surgery: our experience and a review of the literature. $\mathrm{Br} \mathrm{J}$ Neurosurg 2004;18(5):507-512

3 Kim SH, Shin YS, Yoon PH, Kim DI. Emergency endovascular treatment of internal carotid artery injury during a transsphenoidal approach for a pituitary tumor-case report. Yonsei Med J 2002;43(1):119-122

4 Raymond J, Hardy J, Czepko R, Roy D. Arterial injuries in transsphenoidal surgery for pituitary adenoma; the role of angiography and endovascular treatment. AJNR Am J Neuroradiol 1997;18(4): 655-665

5 Ahuja A, Guterman LR, Hopkins LN. Carotid cavernous fistula and false aneurysm of the cavernous carotid artery: complications of transsphenoidal surgery. Neurosurgery 1992;31(4):774-778; discussion 778-779

6 Cabezudo JM, Carrillo R, Vaquero J, Areitio E, Martinez R. Intracavernous aneurysm of the carotid artery following transsphenoidal surgery. Case report. J Neurosurg 1981;54(1):118-121

7 Dusick JR, Esposito F, Malkasian D, Kelly DF. Avoidance of carotid artery injuries in transsphenoidal surgery with the Doppler probe and micro-hook blades. Neurosurgery 2007;60(4, Suppl 2): 322-328; discussion 328-329

8 Ghatge SB, Modi DB. Treatment of ruptured ICA during transsphenoidal surgery. Two different endovascular strategies in two cases. Interv Neuroradiol 2010;16(1):31-37

9 Kocer N, Kizilkilic O, Albayram S, Adaletli I, Kantarci F, Islak C. Treatment of iatrogenic internal carotid artery laceration and carotid cavernous fistula with endovascular stent-graft placement. AJNR Am J Neuroradiol 2002;23(3):442-446

10 Kachhara R, Menon G, Bhattacharya RN, et al. False aneurysm of cavernous carotid artery and carotid cavernous fistula: complications following transsphenoidal surgery. Neurol India 2003;51(1): 81-83

11 Hattori I, Iwasaki K, Horikawa F, Tanji M, Gomi M. [Treatment of a ruptured giant internal carotid artery pseudoaneurysm following transsphenoidal surgery: case report and literature review. [in Japanese]. No Shinkei Geka 2006;34(11):1141-1146 